\title{
Rechtsprechung
}

\section{Zwangsvollstreckung in Kunstgegenstände in der gemeinsamen Ehewohnung}

Landgericht Düsseldorf, Urteil vom 6. November 2015 - 6 O 346/14

1. Die Drittwiderspruchsklage ist auch gegenüber der Vollstreckung von Arresten und einstweiligen Verfügungen statthaft.

2. Die zugunsten der Gläubiger wirkende Vermutungswirkung des § 1362 Abs. 1 S. 1 BGB, wonach im Besitz von Eheleuten stehende Gegenstände dem schuldenden Ehepartner gehören, kann nur durch den vollen Beweis, dass das Eigentum im Miteigentum beider Ehegatten oder des nichtschuldenden Ehegatten steht, entkräftet werden. (Leitsätze der Redaktion)

\section{Tatbestand}

- Gegen den Ehemann der Klägerin, Herrn B9, sowie gegen die von ihm geführten Gesellschaften T2 B5 und B4 erging am 14. Juli 2014 ein Arrestbefehl (Az. 6 O 264/14). Hieraus betreiben die Beklagten die Zwangsvollstreckung in die nunmehr streitgegenständlichen Gegenstände, ein Nagelbild von C, ein Bild von T, sowie zwei Ton-/Terrakottaköpfe, welche sich im von der Klägerin und ihrem Ehemann gemeinsam bewohnten Haus in der $\mathrm{J}$-Straße in $\mathrm{E}$ befanden. Die Beklagten pfändeten diese.

Die Beklagten sind von der Klägerin, die ihr Eigentum hieran behauptet, außergerichtlich zur Freigabe der Pfandgegenstände aufgefordert worden, verweigerten diese aber.

Die Klägerin behauptet, sie habe das Gemälde „Z" zum Kaufpreis von 520.000 Euro von der D erworben, die es wiederum zu einem Preis iHv 500.000 Euro von der $\mathrm{H} \mathrm{GmbH}$ in E erworben habe, welche das Bild wiederum in Kommission vom Künstler gehabt habe und zum Verkauf berechtigt gewesen sei.

Auf die als Anlage K1 zur Akte gereichte Rechnung vom 16. April 2013 habe sie entsprechend des Überweisungsträgers vom 19. April 2013 (Anlage K2) 490.000 Euro gezahlt. Das Geld stamme aus dem Veräußerungserlös ihres Hauses in der H-Straße, welcher ihr in Höhe von 1.127.875,83 Euro am 22. Dezember 2011 gutgeschrieben worden sei. Das Geld sei zunächst als Festgeld angelegt, sowie für Umbaumaßnahmen in der gemeinsamen Immobilie verwendet worden.

Sie habe bereits bei der Eröffnung der Galerie von $\mathrm{H}$ am 9. September 2011 das Gemälde gesehen und sodann überlegt, dieses zur Altersversorgung zu erwerben. Da ihr der Veräußerungserlös ihrer Immobilie noch nicht zur Verfügung gestanden habe, sei die D dazwischengeschaltet worden. Die verbliebene Differenz iHv 30.000 Euro sei ihr gestundet worden. Das Gemälde sei zunächst nach Eingang des Kaufpreises beim Galeristen $\mathrm{H}$ in das zentrale Kunstlager der B5 verbracht und im Anschluss an ihre Zahlung zu ihr geliefert worden. Das Eigentum sei ihr von ihrem Mann als vertretungsberechtigter Geschäftsführer der D übertragen worden.

Ihr Eigentum habe sie auch in ihrem Umfeld kommuniziert. Anschließenden Rückkaufbemühungen des Galeristen, des Zeugen $\mathrm{H}$, sei ihr Mann mit der Begründung entgegengetreten, dass das Werk nicht mehr ihm, sondern nunmehr ihr gehöre. Bei einem gemeinsamen Abendessen im Frühjahr 2013 habe ihr Mann der Ehefrau des Künstlers, der Zeugin V, ebenfalls mitgeteilt, dass er das Bild an seine Frau übereignet habe.

Das Bild aus der Serie "CT-Paintings" von T sei ihr von diesem im Dezember 2012 geschenkt worden, da sie Ateliergespräche des Künstlers moderiert und vorbereitet, sowie einen Festvortrag gehalten habe. Dies wird von Beklagtenseite nach Durchführung der Beweisaufnahme nicht mehr bestritten.

Die zwei streitgegenständlichen Terrakottaköpfe befänden sich bereits seit ihrer Studienzeit in München in ihrem Eigentum, lange bevor sie ihren jetzigen Ehemann kennengelernt habe.

Die Klägerin beantragte ursprünglich,

die von den Beklagten aus dem vollstreckbaren Arrestbefehl in dem Arrestverfahren vor dem Landgericht Düsseldorf vom 14. Juli 2014, Az. 6 O 264/14, betriebene Zwangsvollstreckung in die Kunstobjekte

"Z", Hommage an Roman Opalka, Gemälde von C, 2011, 230 x $230 \mathrm{~cm}$, Nails, latex color on canvas on wood, RN 14344 („Z"),

Bild von T aus der Serie "CT-Paintings", ca. $100 \times 100 \mathrm{~cm}$ (,Painting ${ }^{\prime \prime}$,

zwei Skulpturen, Ton-/Terrakottaköpfe, auf dem Kaminsims („Tonköpfe ${ }^{\prime \prime}$ ) 
für unzulässig zu erklären.

Mit Schriftsatz vom 30. Oktober 2014 erweiterte die Klägerin die Klage um den Hilfsantrag,

die Veräußerung des Kunstobjektes „Z", Hommage an Roman Opalka, Gemälde von C, 2011, 230 x 230 cm, Nails, latex color on canvas on wood, RN 14344 („Z"), im Wege der Zwangsvollstreckung für unzulässig zu erklären.

Die Beklagten beantragten zunächst mit Schriftsatz vom 23. September 2014,

die Klage abzuweisen.

In der mündlichen Verhandlung vom 17. März 2015 haben die Beklagten nach Vernehmung des Zeugen T und Konkretisierung des Klageantrags hinsichtlich des Objekts zu 2.) auf Anlage K17 ein Teilanerkenntnis erklärt. Hierüber ist am 30. März 2015 ein entsprechendes Teilanerkenntnisurteil ergangen, mit welchem die Zwangsvollstreckung in das in Anlage K17 abgebildete Kunstwerk von T aus der Serie "CT-Paintings", ca. $100 \mathrm{x}$

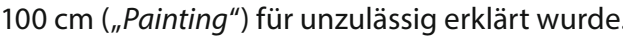

Die Klägerin beantragt nunmehr noch,

die von den Beklagten aus dem vollstreckbaren Arrestbefehl in dem Arrestverfahren vor dem Landgericht Düsseldorf vom 14. Juli 2014, Az. 6 O 264/14, betriebene Zwangsvollstreckung in die Kunstobjekte

"Z", Hommage an Roman Opalka, Gemälde von C, 2011, 230 x $230 \mathrm{~cm}$, Nails, latex color on canvas on wood, RN $14344\left({ }^{\prime \prime} Z^{\prime \prime}\right)$

zwei Skulpturen, Ton-/Terrakottaköpfe, auf dem Kaminsims ("Tonköpfe $\left.{ }^{\prime \prime}\right)$

für unzulässig zu erklären;

hilfsweise,

die Veräußerung des Kunstobjektes „Z", Hommage an Roman Opalka, Gemälde von C, 2011, 230 x 230 cm, Nails, latex color on canvas on wood, RN 14344 („Z"), im Wege der Zwangsvollstreckung für unzulässig zu erklären.

Die Beklagten beantragen weiterhin,

die Klage abzuweisen.

Die Beklagten behaupten, das Werk "Z" sei nicht von der D erworben und anschließend wirksam an die Klägerin übereignet worden. Die Eigentumsverhältnisse hieran seien nicht nachvollziehbar. Dies gehe schon daraus hervor, dass ein Teilbetrag von 250.000 Euro nicht von der D, sondern einer weiteren Gesellschaft des Ehemanns der Klägerin, der B5 Gesellschaft für
Kunstberatung an den Zeugen $\mathrm{H}$ erfolgt sei. Die Bankverbindung, an welche gemäß der Anlagen K1 und K2 die Zahlungen durch die Klägerin erfolgt sein sollen, seien ebenfalls nicht der D, sondern der B4 GmbH zuzuordnen. Aufgrund der Tatsache, dass lediglich 490.000 Euro gezahlt worden sein sollen, sei zudem allenfalls von einem Eigentumsvorbehaltskauf auszugehen.

Ohnehin sei, ein entsprechender Vertrag unterstellt, von einem Scheingeschäft auszugehen.

Eine unbefristete Stundung des Restkaufpreises unterstellt, läge zudem ein kollusives und bewusst zum Nachteil der D erfolgtes Zusammenwirken vor, welches zur Unwirksamkeit des Rechtsgeschäftes führe.

Letztlich fehle es auch an einem vollständigen Besitzverlust, folglich an einer Übergabe, da der Zeuge B4 als Organ der Geschäftsführerin der vermeintlich veräußernden $D$ weiterhin im Mitbesitz des Werkes geblieben sei.

Wegen des weiteren Vorbringens der Parteien wird auf den Inhalt der gewechselten Schriftsätze, sowie der zur Akte gereichten Anlagen Bezug genommen.

Die Kammer hat aufgrund Beweisbeschlusses vom 16. Dezember 2014 Beweis erhoben über den Eigentumserwerb des Werks " $Z$ " durch die D vom Galeristen $\mathrm{H}$ und die Übereignung von der $\mathrm{D}$ an die Klägerin, die Übergabe des Werkes, sowie die Behauptung, die Klägerin und ihr Ehemann haben gegenüber der Zeugin V bestätigt, dass es sich um ihr Werk handele durch Vernehmung der Zeugen H, B9, V, N und F. Des Weiteren wurde Beweis erhoben über den Eigentumserwerb an dem Bild aus der Serie "CT-Paintings" vom Künstler T durch dessen Vernehmung und ihr Eigentum an den streitgegenständlichen Terrakottaköpfen durch Vernehmung des Zeugen F.

\section{Entscheidungsgründe}

Die Klage ist zulässig, jedoch nur in tenoriertem Umfang begründet. Bezüglich des streitgegenständlichen Kunstwerks „Z" ist die Klage sowohl hinsichtlich des gestellten Hauptantrags als auch des Hilfsantrags unbegründet.

I.

Die in der Hauptsache vorliegend erhobene Drittwiderspruchsklage ist statthaft. Sie ist insbesondere auch gegenüber der Vollstreckung von Arresten und einstweiligen Verfügungen statthaft (MünchKomm-Schmidt/Brinkmann, ZPO, 4. Aufl., 2012, $\S 771, \mathrm{Rn} .7)$ 
II.

Die Drittwiderspruchsklage ist bezüglich der gepfändeten Terrakottaköpfe begründet, hinsichtlich des Werkes „Z" indes unbegründet.

1. Die Drittwiderspruchsklage ist begründet, wenn der Klägerin der ihr obliegende Nachweis gelingt, dass ihr an den streitgegenständlichen Kunstgegenständen ein die Veräußerung hinderndes Recht zusteht. Insoweit macht die Klägerin Alleineigentum geltend.

Dabei wird gemäß § 1362 Abs. 1 S. 1 BGB zu Gunsten der Gläubiger des Ehemannes vermutet, dass die im Besitz eines Ehegatten oder beider Ehegatten befindlichen beweglichen Sachen dem Schuldner, vorliegend also dem Ehemann der Klägerin gehören. Vorliegend befanden sich sämtliche Kunstgegenstände in der gemeinsam bewohnten Wohnung in der J-Straße in E, sodass die Vermutungswirkung des § 1362 Abs. 1 S. 1 BGB von der Klägerin entkräftet werden muss.

Die Vermutung wird nur widerlegt durch den vollen Beweis, dass die streitbefangenen Gegenstände im Miteigentum beider Gatten stehen oder dass der nichtschuldende Gatte Alleineigentümer ist (Staudinger-Voppel, BGB-Neubearbeitung, 2012, $\S 1362$ Rn. 43, 45; BeckOK BGB-Beutler, Stand: 1.8.2015, § 1362 Rn. 3).

Der hierzu erforderliche Nachweis ihres Alleineigentums ist der Klägerin indes nur bezüglich der Terrakottaköpfe gelungen.

2. Aufgrund der durchgeführten Beweisaufnahme geht die Kammer von der Eigentümerstellung der Klägerin an den Terrakottaköpfen, wie sie sich auf dem als Anlage K18 zur Akte gereichten Lichtbild darstellen, aus.

Bei seiner Vernehmung in der mündlichen Verhandlung vom 17. März 2015 gab der Zeuge F hierzu glaubhaft an, dass er sich erinnern könne, in dem Studentenzimmer der Klägerin auf der Q-Straße in L unter anderem afrikanische Masken und Terrakottaköpfe gesehen zu haben. Dies begründete er nachvollziehbar mit einem engen freundschaftlichen Verhältnis zur Klägerin, was dazu führte, dass er mehrfach in ihrer Wohnung gewesen sei. Seine Erinnerungen habe er anhand privatschriftlicher Notizen auffrischen können, die er seit seinem 17. Lebensjahr führe. An der Glaubhaftigkeit der Aussage bestehen keine Bedenken.

Zwar konnte der Zeuge lediglich bestätigen, dass die von inm in Erinnerung befindlichen Terrakottaköpfe so ähnlich ausgesehen haben, wie auf dem Lichtbild in Anlage K 18 und er sich genauer nicht mehr an die einzelnen Terrakottaköpfe und deren Aussehen erinnern könne. Dies ist für die Kammer indes ausreichend, um den für das praktische Leben brauchbaren Grad an Gewissheit von der Eigentümerstellung der Klägerin, die Zweifeln Einhalt gebietet, ohne sie vollständig auszuschließen, zu verschaffen.
Die Kammer erachtet es als naheliegend, dass die streitgegenständlichen Terrakottaköpfe auch diejenigen sind, welche der Klägerin bereits zu ihrer Studienzeit gehörten, es sich jedenfalls um ihr Eigentum handelt. Die Anforderungen an eine glaubhafte Aussage dürften hier nicht dergestalt überspitzt werden, dass nach Ablauf von rund 25 Jahren eine mit hundertprozentiger Sicherheit erfolgende Identifizierung verlangt wird. Zwar ist es aufgrund der Aussage des Zeugen auch durchaus möglich, dass die Terrakottaköpfe, die der Zeuge in Erinnerung hat, nicht (mehr) diejenigen sind, die nunmehr streitgegenständlich sind. Jedoch ist aus der Aussage eben auch zu entnehmen, dass die Klägerin bereits früher Interesse an derartigen, von den ansonsten streitgegenständlichen Gemälden abweichenden, Kunstobjekten hatte, sodass davon auszugehen ist, dass die streitgegenständlichen Köpfe, selbst wenn es sich nicht mehr um dieselben Köpfe wie im Jahre 1990 handelt, dennoch der Klägerin gehören.

3. Vom Eigentum der Klägerin am Nagelbild " $Z$ " des Künstlers $C$ ist die Kammer aufgrund der durchgeführten Beweisaufnahme indes nicht überzeugt.

Die hierzu vernommenen Zeugen konnten die sich aus den sonstigen Umständen ergebenden Zweifel am Eigentumserwerb der Klägerin nicht beseitigen, vielmehr sind die Aussagen der Zeugen selbst Widersprüchen und Zweifeln ausgesetzt.

a) Zweifel an dem behaupteten Eigentumserwerb durch die Klägerin ergeben sich bereits daraus, dass die zum Zwischenerwerb des Werks durch die D vorgetragenen Beweggründe fragwürdig erscheinen.

Es ist zum einen nach wie vor nicht nachvollziehbar, wieso die Klägerin, auf deren Girokonto am 22. Dezember 2011 ein Betrag in Höhe von 1.127.875,83 Euro gutgeschrieben worden sein soll, hiervon nicht den vollständigen Kaufpreis, sondern lediglich anderthalb Jahre später 490.000 Euro gezahlt hat und bezüglich des Restbetrages von 30.000 Euro eine offensichtlich unbefristete Stundung der Kunsthandelsgesellschaft in Anspruch nehmen musste.

Die Klägerin ließ hierzu zunächst vortragen, dass das Geld vorerst als Festgeld angelegt worden sei. Dem steht bereits die Aussage des Zeugen B4 entgegen, der aussagte, dass "eine Anlage dieses Geldbetrages in Festgeld [...] nicht rentabel" gewesen sei.

Sodann trägt die Klägerin hierzu zwar weiter vor, sie habe den Verkaufserlös „zunächst vorrangig“ zur Finanzierung von Renovierungsarbeiten an der J-Straße verwandt. Wann die einzelnen Arbeiten durchgeführt wurden, mit welchem Kostenaufwand gerechnet wurde und wie hoch die Kosten letztlich waren, wird indes nicht dargelegt. Es stellt sich daher weiterhin die Frage, wieso der Klägerin bereits beim Erwerb des Werkes durch die D bekannt war, dass sie sich das Werk werde leisten können, wenn selbst bei Verfügbarkeit des Geldes Ende 2011 
noch nicht bezahlt wurde, sondern erst - offensichtlich nach Durchführung der Renovierung - ein Teilbetrag von 490.000 Euro gezahlt wurde. Wenn die Klägerin noch nicht absehen konnte, wie viel Geld sie für die Renovierung benötigte, ist nicht nachvollziehbar, wieso sie dann aber schon davon ausging, sich das Gemälde überhaupt leisten zu können.

Des Weiteren erscheint das gewählte Konstrukt des Zwischenerwerbs auch aufgrund des jeweiligen Kaufpreises fragwürdig. Zwar ist es durchaus nachvollziehbar, dass der D ein Zuschlag von 20.000 Euro auf den eigentlichen Kaufpreis zugebilligt wird, auch wenn es sich hier um ein Geschäft unter Beteiligung von Eheleuten handelt, da die D nicht ausschließlich zum Vermögen des Ehemanns der Klägerin gehört und vorliegend bei einem bloßen Zwischenerwerb anderenfalls einen Betrag von 500.000 Euro faktisch zinsfrei zur Verfügung stellen würde. Wenn dann anschließend jedoch nur ein Teilbetrag von 490.000 Euro gezahlt und der Restbetrag, wobei bereits nicht vorgetragen wurde, wieso der prozentual geringfügige Restbetrag bis zuletzt nicht bezahlt wurde, auf unbestimmte Zeit gestundet wird, verliert der zugunsten der D gedachte Vorteil schlussendlich an Wert.

Letztlich sind auch die Angaben zum genauen Zeitpunkt der Eigentumsübertragung seitens der $\mathrm{D}$ an die Klägerin widersprüchlich. Während in der Klageschrift auf Rechnungsdatum und Überweisung im April 2013 abgestellt wird, ist im Schriftsatz vom 30. Oktober 2014 von einer Übereignung bereits im März 2012 die Rede, mithin mehr als ein Jahr vor der Rechnungsstellung und Bezahlung. Auch dieses Datum bleibt im Hinblick auf die Aussage des Zeugen $\mathrm{H}$ widersprüchlich, der insoweit angab, bereits im Jahr 2011 vom Zeugen B4 die Information erhalten zu haben, das Werk gehöre nunmehr der Klägerin.

b) Diese Widersprüche konnten durch die hierzu eingeholten Zeugenaussagen nicht dergestalt beseitigt werden, dass die Kammer von der Eigentümerstellung der Klägerin überzeugt ist.

(1) Die Aussagen der Zeugen N und A waren für die dingliche Einigung zwischen der Klägerin und ihrem Ehemann als Geschäftsführer der D unergiebig.

Sie haben zwar ausgesagt, dass das Werk " $Z$ " vom Galeristen $\mathrm{H}$ in das Lager der $\mathrm{D}$ und von dort einige Zeit später in die Privatwohnung der Klägerin verbracht worden ist. Dies sei auf Veranlassung des Zeugen B4 erfolgt. Hintergründe seien ihnen indes nicht mitgeteilt worden.

Die Verbringung des Bildes in das gemeinsame Haus der Klägerin und ihres Mannes, begründet indes für sich noch keine zwingende Schlussfolgerung dahingehend, dass dies der Übergabeakt einer Übereignung der D an die Klägerin darstellt, zumal auch beide Ehegatten das Bild in Empfang genommen haben.
(2) Der Zeuge B4 bestätigte den klägerischen Vortrag zur Eigentumsübertragung an die Klägerin zwar insgesamt. Jedoch sind die bereits genannten Widersprüche zur Kaufpreiszahlung verblieben. Des Weiteren war die Aussage in weiteren Punkten widersprüchlich, teilweise nicht nachvollziehbar, im Ergebnis jedenfalls erheblichen Bedenken ausgesetzt.

So schilderte der Zeuge zunächst, das Bild für eine seiner Firmen erworben zu haben. Die Klägerin habe sich erst „im nächsten Jahr" entschieden, dass sie das Exponat gerne erwerben würde. Obwohl der Erwerb durch die Klägerin hier noch als unsicher dargestellt wurde, gab der Zeuge an, dass man Gründe für einen erforderlichen Zwischenerwerb durch die D gehabt habe. So sei das Grundstück auf der H-Straße zwar bereits verkauft, der Kaufpreis aber noch nicht geflossen, weswegen die $D$ zwischengeschaltet werden sollte. Dies wiederum macht nur Sinn, wenn von Anfang an ein Erwerb durch die Klägerin intendiert gewesen ist.

Weiter gab der Zeuge an, dass der Kaufpreis an sie um 20.000 Euro zugunsten der D erhöht worden sei, jedoch iHv 30.000 Euro auf unbestimmte Zeit gestundet wurde, da die Klägerin dieses Geld noch für Umbauarbeiten zurückhalten wollte. Es sei noch unklar gewesen, wie viel Geld eine Renovierung des Hauses verbrauchen wird. Auf der anderen Seite sei aber "zu keiner Zeit die Rede davon gewesen, dass die Klägerin den Kaufpreis nicht mehr zahlen können werde". Dieser Vortrag erscheint absolut ins Blaue hinein. Die Klägerin erhielt durch die Veräußerung des Objekts E T3 am 22. Dezember 2011 einen Betrag in Höhe von 1.127.875,83 Euro. Wie bereits ausgeführt und vom Zeugen auch nicht weiter unterlegt, ist nicht ersichtlich, ob zur Zeit der behaupteten Kaufpreiszahlung der Klägerin im April 2013 die behaupteten Umbaumaßnahmen bereits fertig waren und nur noch kleinere Arbeiten ausstanden, die einen Einbehalt der gestundeten 30.000 Euro rechtfertigt oder ob möglicherweise noch gar nicht begonnen wurde. Überhaupt ist weder dem klägerischen Vorbringen, noch den Angaben des Zeugen nachvollziehbar zu entnehmen, mit welchen Beträgen man bezüglich des Umbaus kalkuliert hat und wieso man bei einem verfügbaren Betrag von 1,1 Mio. Euro davon ausging, dieses Geld einerseits für die Renovierung zu benötigen, andererseits sicher sein konnte, dass die Klägerin den Kaufpreis schlussendlich bezahlen werde können.

(3) Des Weiteren steht die Aussage des Zeugen B4 bezüglich einzelner Punkte im Widerspruch zur Aussage des Zeugen $\mathrm{H}_{\text {, }}$ die bereits an sich ebenfalls Zweifel offenlässt.

So bestätigte der Zeuge $\mathrm{H}$ zwar, dass der Zeuge B4 das Bild erworben und anschließend auf seine Nachfragen wegen weiterer Interessenten geäußert habe, dass das Bild nunmehr seiner Frau, der Klägerin, gehöre. Jedoch weicht die Aussage in nicht unerheblicher Weise von den Schilderungen des Zeugen B4 ab. 
Dieser gab an, dass er mit dem Zeugen $\mathrm{H}$ in Gegenwart der Klägerin auf der Eröffnung von dessen Galerie über das Bild gesprochen habe. Der Zeuge $\mathrm{H}$, der angab, dass die Galerieeröffnung ein persönlicher Höhepunkt seines Lebens und das Bild " $Z$ " das bedeutendste Exponat der Ausstellung gewesen sei, konnte sich indes nicht mehr erinnern, ob die Klägerin bei der Eröffnung überhaupt anwesend war, bezüglich des Zeugen B4 verneinte er sogar eine Anwesenheit.

Zu seinen späteren Nachfragen, ob der Zeuge B4 das Bild wieder verkaufen wolle, äußerte sich der Zeuge sodann sehr zielorientiert auch ohne gerichtliches Befragen. Seine Aussage konzentrierte sich selbständig auf den wesentlichen Punkt, dass der Zeuge B4 ihm gegenüber gesagt habe „Das Bild gehöre jetzt der $O^{\prime \prime}$, sodass die Aussage zumindest insoweit vorbereitet wirkte.

Abweichungen ergaben sich diesbezüglich zudem darin, dass der Zeuge $\mathrm{H}$ sogar zwei weitere Nachfragen beim Zeugen B4 schilderte, dieser aber nur eine Anfrage. Vor allem ergibt sich, wie bereits ausgeführt, ein wesentlicher Widerspruch aber auch daraus, dass der Zeuge U von einer Anfrage noch im Jahr 2011 berichtete, auf die ihm ebenfalls erwidert worden sei, das Bild gehöre jetzt der Klägerin, obwohl nach eigenem Vorbringen der Klägerin eine Übereignung frühestens 2012 erfolgt sein soll.

Eine weitere wesentliche Abweichung der Aussagen liegt aber auch darin, dass der Zeuge $\mathrm{H}$ den Vortrag des Zeugen B4 dahingehend nicht bestätigte, dass er auf eine schnelle Zahlung des Kaufpreises bestanden habe. Der Zeuge B4 begründete das Erfordernis der Zwischenschaltung der D gerade damit, dass der Zeuge $\mathrm{H}$ das Geld schnell haben wollte, der Erlös aus dem Hausverkauf bei der Klägerin aber noch nicht verfügbar gewesen sei, weswegen eben die D zwischengeschaltet werden musste. Dies verneinte der Zeuge $\mathrm{H}$. Eine besondere Eilbedürftigkeit wies er zurück, es seien übliche Zahlungsfristen vereinbart worden.

(4) Letztlich hat auch die Aussage der Ehefrau des Künstlers, V, die Kammer nicht von einem Eigentumserwerb der Klägerin überzeugen können.

Zwar bestätigte auch die Zeugin den insoweit von Klägerseite erbrachten Vortrag dahingehend, dass im Frühjahr 2013 anlässlich eines Besuches der Zeugin bei der Klägerin ihr gegenüber angegeben worden sein soll, dass das Bild nunmehr der Klägerin gehöre, jedoch bestehen auch insoweit Bedenken an der Glaubhaftigkeit dieser Aussage.

Zunächst erfolgte auch die, im Übrigen detailarme Aussage der Zeugin zielgerichtet auf die Beweisfrage, die nahezu wörtlich bestätigt worden ist. Dies begegnet insbesondere deshalb Bedenken, da die Zeugin sich nicht einmal erinnern konnte, wer an dem gemeinsamen Abendessen teilgenommen hat, auf der anderen Seite allerdings wörtlich wiedergeben konnte, was zu dem Bild gesagt worden sein soll.
Des Weiteren erachtet es die Kammer zumindest als ungewöhnlich, dass, wie von der Zeugin geschildert, die erste Aussage der Klägerin, angesprochen auf das Werk, gewesen sein soll, dass sie das Bild von ihrem eigenen Geld gekauft habe und es ihr gehöre. Das Erfordernis der von der Zeugin geschilderten besonderen Betonung durch die Klägerin, das Bild von ihrem "eigenen Geld" erworben zu haben, erschließt sich der Kammer auch nach ergänzender Befragung der Zeugin nicht.

c) Im Ergebnis ist die Kammer daher nicht davon überzeugt, dass das streitgegenständliche Werk " $Z$ " tatsächlich von der Klägerin zu Alleineigentum von der D, deren Erwerb dahinstehen kann, erworben wurde.

Die Kammer sieht es weder als erwiesen an, dass eine wirksame unbedingte Übereignung von der $D$ an die Klägerin durch den Zeugen B4 erfolgt ist, noch dass dieser Eigentumserwerb anschließend gegenüber den Zeugen $\mathrm{H}$ und $\mathrm{V}$ auch kommuniziert worden ist.

Die Kammer ist dementsprechend auch nicht von einer auf die vollständige Bezahlung des behaupteten Kaufpreises von 520.000 Euro bedingten Übereignung, die schlussendlich nicht einmal von Klägerseite behauptet wird, und somit einem Erwerb eines Anwartschaftsrechtes auf Seiten der Klägerin überzeugt, sodass die Klage auch hinsichtlich des gestellten Hilfsantrags abzuweisen war. (Entscheidung von der Redaktion bearbeitet.) 